\title{
Racial Disparities in Patients with Melanoma: A Multivariate Survival Analysis
}

\author{
Joshua Brady' \\ Reem Kashlan' \\ Julie Ruterbusch ${ }^{2}$ \\ Mehdi Farshchian (1D) ${ }^{3}$ \\ Meena Moossavi ${ }^{3}$ \\ 'Wayne State University School of \\ Medicine, Detroit, MI, USA; ${ }^{2}$ Department \\ of Oncology, Wayne State University, \\ Detroit, MI, USA; ${ }^{3}$ Department of \\ Dermatology, Wayne State University, \\ Detroit, MI, USA
}

Purpose: As the most common cause of skin cancer death, incidence and mortality of melanoma vary widely between ethnic and racial groups.

Methods: Surveillance, Epidemiology, and End Results (SEER) data were used to examine the incidence and survival in patients with melanoma concerning race and ethnicity in Wayne County, Michigan between 2000 and 2016.

Results: Analysis of data revealed significantly higher melanoma-specific death in nonHispanic black patients compared to their non-Hispanic white counterparts $(p<0.001)$. However, no increased risk of death due to melanoma was observed following adjustment of data for the stage, age, and sex (H.R. $=1.00,95 \%$ CI 0.64-1.56).

Conclusion: Non-Hispanic black patients have the highest percentage of late-stage melanoma. Increased incidence of melanoma mortality in non-Hispanic black patients is likely a consequence of late-stage diagnosis.

Keywords: melanoma, racial disparities, epidemiology, clinical research, skin of color

\section{Introduction}

Melanoma accounts for $75 \%$ of all skin cancer deaths in the United States. ${ }^{1}$ In 2019, there were an estimated 96,480 new melanoma cases in the United States, with an estimated 7,230 deaths. ${ }^{2}$ In general, cancer incidence and mortality of all types vary widely between ethnic and racial groups due to disparities in wealth and socioeconomic status. These inequalities lead to differences in cancer prevention/ treatment and increases in exposures to several risk factors, such as dietary, smoking, and delays in screening patterns. ${ }^{2}$

The overall cancer incidence rate in non-Hispanic black (NHB) men was 9\% higher than non-Hispanic white (NHW) men in data collected from 2011 to 2015. In men and women combined, the black-white disparity in overall cancer mortality has declined to $14 \%$ in 2016 (183.6 vs 160.7 per 100,000 population). ${ }^{2}$ The narrowing racial gap in cancer mortality over the past several decades is promising. However, socioeconomic inequalities are widening as the years go on, causing people who live in counties with higher poverty levels to carry the heaviest burden of preventable cancers, such as melanoma. ${ }^{2}$ Overall cancer death rate was estimated to be $20 \%$ higher among the most impoverished counties than the more affluent counties. ${ }^{2}$

Although individuals of lighter skin tones are more likely to develop melanoma, studies have found that African American populations have a worse prognosis than Caucasian individuals. ${ }^{3}$ It has been found that NHW patients had the highest incidence rate at 45.8 diagnoses of melanoma per 100,000 compared to NHB patients $(1.35){ }^{3}$
Correspondence: Mehdi Farshchian Department of Dermatology, Wayne State University, I8100 Oakwood Blvd., Suite 300, Dearborn, MI, 48I24

Email mfarshch@med.wayne.edu 
However, stage, age, and race showed significantly shorter survival for black patients than their white counterparts, with acral lentiginous melanoma being most common in NHB patients. ${ }^{3,4}$ According to the United States Census Bureau, Wayne County, Michigan, has a population of 1,749,343 and a $19.8 \%$ poverty rate, compared to the $10.5 \%$ national rate. ${ }^{5}$ The entire county has a NHW population of $54.6 \%$ and a NHB population of $38.7 \%$. The largest city within Wayne County, and the state of Michigan as a whole, is Detroit, which has a population of 670,031 and carries the highest burden of poverty at $35 \%$. Detroit itself is comprised of $14.7 \% \mathrm{NHW}$ individuals and $78.3 \%$ NHB individuals. ${ }^{5}$ In this study, Surveillance, Epidemiology, and End Results (SEER) data were used to examine the incidence and survival in patients with melanoma concerning race in Wayne County, Michigan.

\section{Methods}

Primary malignant melanoma of the skin cases diagnosed in 2000-2016 was identified using the SEER public use dataset. Inclusion criteria consisted of living in Wayne County, Michigan, where the state's largest city of Detroit is located, at the time of diagnosis. Additional criteria included diagnostic confirmation by positive histology, known age, and known survival time. For this analysis, race/ethnicity other than non-Hispanic white and non-Hispanic black were excluded $(\mathrm{N}=129)$, resulting in 3,540 cases included in this report. Age-adjusted incidence rates by race/ethnicity category along with rate ratios and $95 \%$ confidence intervals were calculated using SEER*Stat software. The distribution of sex, age at diagnosis, SEER summary stage, and vital status by race/ethnicity were compared using a general chi-square test. Survival time was calculated in months from diagnosis to death or last follow-up. The cumulative incidence of death due to melanoma and death due to other causes was computed using the Fine and Gray method. The differences by race/ethnicity were compared using Gray's test. Proportional hazards regression was used to estimate hazard ratios and 95\% confidence intervals for melanomarelated and other cause mortality. Both univariate and multivariate estimates were calculated, and the multivariate model included covariates selected a priori: sex, age at diagnosis, and SEER summary stage. To test the proportionality assumption, an interaction term between race/ ethnicity and the log of survival time was included in both the univariate and multivariate models.

\section{Results}

The demographic distribution of both groups is summarized in Table 1. Age and stage of melanoma at diagnosis were significantly different between NHB and NHW patients (Table 1). As outlined by the competing risk analysis in Figure 1, NHB patients were more likely to have a statistically significant (Gray's test $\mathrm{p}<0.001$ ) increase in melanoma-specific death compared to their NHW counterparts. An unadjusted univariate proportional hazards regression illustrated a hazard of death due to melanoma to be 3.24 (95\% CI 2.11-4.99). However, when adjusted for the stage, age, and sex, there was no longer an increased risk of death due to melanoma (H.R. $=1.00,95 \%$ CI $0.64-1.56)$. These data suggest that the increased incidence of mortality from

Table I Characteristics of Cutaneous Melanoma Cases Diagnosed 2000-2016 in Wayne County, Michigan for NonHispanic Black (NHB) and Non-Hispanic White (NHW) Patients

\begin{tabular}{|l|c|c|c|c|c|}
\hline & \multicolumn{2}{|c|}{ NHW } & \multicolumn{2}{|c|}{ NHB } & p-value \\
\hline & N & $\%$ & N & $\%$ & \\
\hline Total & 3,467 & & 73 & & \\
\hline Sex & & & & & $<0.001$ \\
\hline Male & 1,945 & $56 \%$ & 22 & $30 \%$ & \\
\hline Female & 1,522 & $44 \%$ & 51 & $70 \%$ & \\
\hline Age at Diagnosis & & & & & 0.258 \\
\hline$<50$ & 1,171 & $34 \%$ & 19 & $26 \%$ & \\
\hline $50-59$ & 739 & $21 \%$ & 15 & $21 \%$ & \\
\hline $60-69$ & 660 & $19 \%$ & 19 & $26 \%$ & \\
\hline $70-79$ & 544 & $16 \%$ & 9 & $12 \%$ & \\
\hline $80+$ & 353 & $10 \%$ & 11 & $15 \%$ & \\
\hline SEER* Summary Stage & & & & & $<0.001$ \\
\hline Local & 2,935 & $86 \%$ & 38 & $55 \%$ & \\
\hline Regional & 328 & $10 \%$ & 17 & $25 \%$ & \\
\hline Distant & 138 & $4 \%$ & 14 & $20 \%$ & \\
\hline Unknown/Unstaged & 66 & - & 4 & - & \\
\hline Vital Status & 376 & $11 \%$ & 22 & $30 \%$ & \\
\hline Alive & $13 \%$ & 11 & $15 \%$ & \\
\hline Death due to melanoma & & & & $<0.001$ \\
\hline Death due to other causes & 441 & $76 \%$ & 40 & $55 \%$ & \\
\hline
\end{tabular}

Note: *Surveillance, Epidemiology, and End Results Database. 


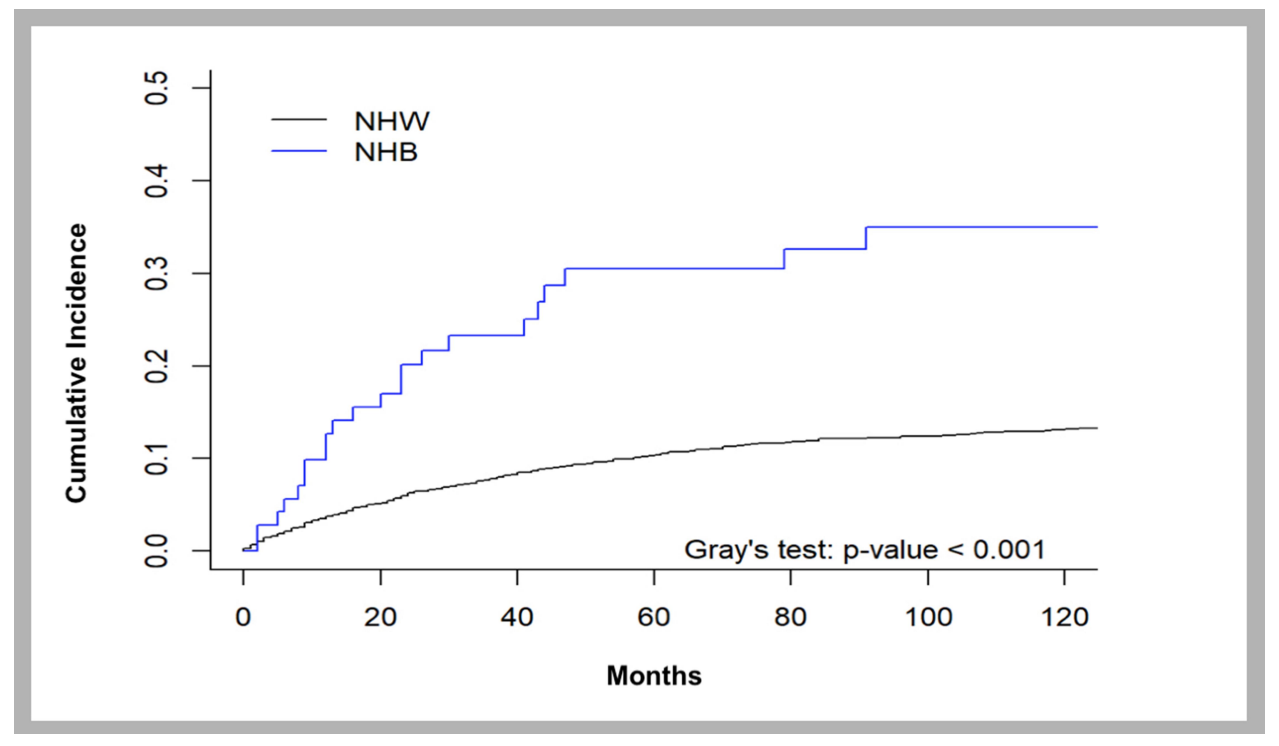

Figure I Competing risk of death due to melanoma in non-Hispanic Black (NHB) and non-Hispanic white (NHW) patients.

melanoma for NHB results from the late stage at diagnosis, rather than any other variables between patients.

\section{Discussion}

Our study echoes previous literature demonstrating that although white patients had a higher incidence rate of melanoma, black patients had the highest percentage of late-stage diagnoses, thereby leading to higher mortality. ${ }^{3}$ Previous multivariate analyses, controlling for age, gender, race, and stage, demonstrated that only gender and stage of disease were significantly associated with survival-thereby supporting our data that race itself is not associated with survival, but the connection of race with stage at diagnosis is crucial to address. ${ }^{6}$ In our statistical analysis, there was an overt difference in melanoma-specific mortality between races; however, once stage was adjusted for, there was no longer a difference in mortality between NHB and NHW patients. This allows us to conclude that the intersection of race and melanoma mortality is dependent on differing stages at diagnosis between the two races. Therefore, it can be posited that the racial disparity surrounding melanoma mortality can be attributed to underlying social factors leading to delayed diagnosis (ie, later-stage melanoma at first presentation) and delayed treatment of NHB patients. Patients with lower SES have trouble accessing care, which needs to be addressed in order to create positive change in disadvantaged cities. The literature has shown that living in a poor neighborhood was associated with a $5 \%$ increase in difficulty in accessing primary care, which is often the first step in being referred to dermatology-specific health care. ${ }^{7}$ Low-income, racial minority, and uninsured individuals continue to be less likely to have an identifiable usual source of care for their healthcare needs. Community health centers (CHCs) have been found to help fill the void of healthcare delivery in underserved areas, by providing comprehensive, coordinated, and culturally sensitive care to underserved patients. ${ }^{7}$ Events such as physician-sponsored community skin cancer screening events can be an easy way to engage target populations while educating them at the same time; these may be at local CHCs or other centrally located, easily accessible public spaces.

It has been found that NHB patients possess a lower self-perceived risk for melanoma and may have less awareness about melanoma, causing these patients to minimize the need for annual full body skin exams. ${ }^{8}$ Additionally, the preponderance of lesions in acral locations and the tendency to overlook a darker lesion on skin of color individuals may be an additional explanation for more late-stage diagnoses leading to the higher mortality rate. ${ }^{6}$ This demonstrates the need for more thorough full body skin exams in patients of color, with an emphasis on acral areas; this starts with changing the narrative surrounding the perceived low risk of skin cancer in patients with skin of color.

Past research also has not shown that melanoma's biological behavior in African-American skin has contributed to the disparity of outcomes, supporting our data that reduced access to care and lower SES in NHB patients in cities like the greater Detroit area are key reasons for the racial disparity of melanoma stage and mortality. ${ }^{6}$ Since overall studies looking at specific vulnerable cities are 
lacking, this analysis regarding health disparities in Wayne County, Michigan, can be used as a surrogate for additional areas with similar socioeconomic profiles around the country. The overall need for increased focus on skin of color education in dermatology residency programs nationwide is evident, but especially for providers practicing in more diverse populations and areas with low SES. It would be prudent for future research to focus on prompt access to care for NHB melanoma patients and specific social obstacles that can be addressed in order to bridge the gap of late-stage diagnoses. This would thereby lessen the burden of racial disparities that is being seen in counties carrying a high percentage of patients with skin of color. By addressing the intersection of social factors and skin cancer, targeted interventions may improve the detection and management of melanoma in this population. Standardized protocols and local educational programs for patients and physicians alike should be set in place to attempt to alleviate racial disparities, especially within more vulnerable communities, such as those that make up Wayne County.

\section{Ethics Statement}

Consent requirement was waived for this study as it is based on a publicly available database with anonymized and confidential patient information.

\section{Funding}

There is no funding to report.

\section{Disclosure}

The authors report no conflicts of interest in this work.

\section{References}

1. Glazer AM, Winkelmann RR, Farberg AS, Rigel DS. Analysis of trends in U.S. melanoma incidence and mortality. JAMA Dermatol. 2017;153(2):225. doi:10.1001/jamadermatol.2016.4512

2. Siegel RL, Miller KD, Jemal A. Cancer statistics, 2019. CA Cancer J Clin. 2019;69(1):7-34. doi:10.3322/caac.21551

3. Dawes SM, Tsai S, Gittleman H, Barnholtz-Sloan JS, Bordeaux JS. Racial disparities in melanoma survival. $J$ Am Acad Dermatol. 2016;75(5):983-991. doi:10.1016/j.jaad.2016.06.006

4. Culp MB, Lunsford NB. Melanoma among non-Hispanic Black Americans. Prev Chronic Dis. 2019;16:180640. doi:10.5888/ pcd16.180640

5. U.S. Census Bureau Quickfacts: Wayne County, Michigan. Available from: https://www.census.gov/quickfacts/waynecountymichigan. Accessed May 10, 2021.

6. Wang Y, Zhao Y, Ma S. Racial differences in six major subtypes of melanoma: descriptive epidemiology. BMC Cancer. 2016;16(1):691. doi:10.1186/S12885-016-2747-6

7. Hussein M, Diez Roux AV, Field RI. Neighborhood socioeconomic status and primary health care: usual points of access and temporal trends in a major US urban area. $J$ Urban Health. 2016;93 (6):1027-1045. doi:10.1007/s11524-016-0085-2

8. Harvey VM, Patel H, Sandhu S, Flynt Wallington S, Hinds G. Social determinants of racial and ethnic disparities in cutaneous melanoma outcomes. Cancer Control. 2014;21(4):343-349. doi:10.1177/ 107327481402100411
Clinical, Cosmetic and Investigational Dermatology is an international, peer-reviewed, open access, online journal that focuses on the latest clinical and experimental research in all aspects of skin disease and cosmetic interventions. This journal is indexed on CAS.
The manuscript management system is completely online and includes a very quick and fair peer-review system, which is all easy to use. Visit http://www.dovepress.com/testimonials.php to read real quotes from published authors. 